\title{
HIGHLIGHTS
}

SURGERY

\section{Laparoscopy versus laparotomy in early-stage endometrial cancer}

The standard treatment for early-stage endometrial cancer is total abdominal hysterectomy (TAH), generally carried out through laparotomy, with bilateral salpingo-oophorectomy. Although this method is effective, the surgical procedure can be associated with considerable morbidity, because of a high incidence of obesity and other comorbidities seen in patients with endometrial cancer.

Compared with TAH, total laparoscopic hysterectomy (TLH) offers a less-invasive treatment approach and has been associated with a reduction in complications. Few randomized studies have compared the two techniques in patients with earlystage endometrial cancer and none has investigated morbidity. Now, two studies report improved quality-of-life and health outcomes and reduced complications in this population of patients treated with TLH compared with TAH.

Mourits and colleagues randomly assigned patients with stage I endometrial cancer to treatment with either TAH $(n=96)$ or TLH $(n=187)$ in 21 medical centers in the Netherlands. The team assessed the occurrence of major complications (intraoperatively and postoperatively) and minor complications, the treatment-related outcomes, and the quality-of-life in both groups.

No marked difference in the incidence of major complications or minor complications was reported between the treatment groups. However, patients treated with TLH experienced benefits that were less pronounced in the TAH group. "The TLH was associated with significantly less blood loss, less use of pain medication, a shorter hospital stay, and a faster recovery," comments lead researcher Claudia Bijen.

In the second trial, Janda and colleagues treated 332 patients with stage I endometrial cancer (recruited from 19 tertiary gynecological centers in New Zealand, Australia, and Hong Kong) with either TAH $(n=142)$ or TLH $(n=190)$. Quality-of-life outcomes were assessed for both surgical techniques during the early postoperative period (up to 4 weeks after surgery) and late postoperative period (up to 6 months after surgery).

"Quality of life was significantly better in patients who had a TLH compared with TAH," reports study chair Andreas Obermair, of the Queensland Centre for Gynaecological Cancer, Australia. This quality-of-life advantage was apparent in the early postoperative period, and was maintained for up to 6 months after surgery. Although interoperative complications were comparable between the two surgical approaches, Obermair highlights that "postoperative adverse events were approximately half in the TLH group compared with the TAH group."

Both studies employed experienced surgeons to carry out TLH. "One of the

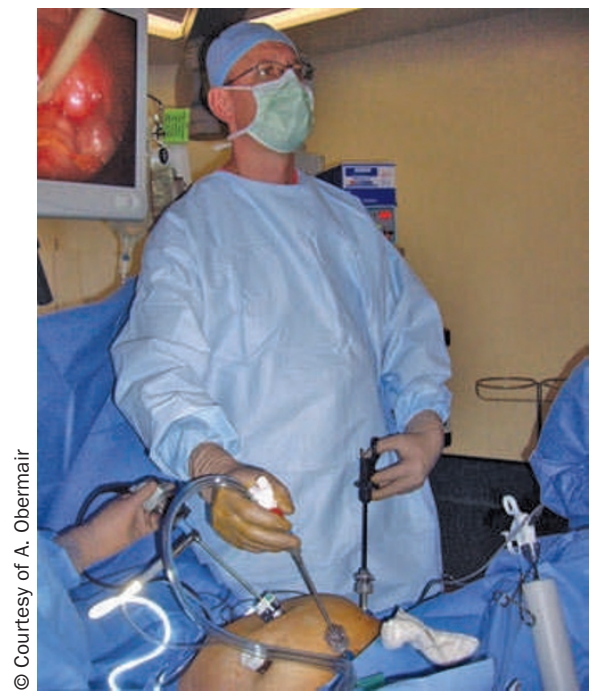

reasons why complications were so low in the TLH group is because all surgeons underwent an accreditation process and all patients had surgery with one of those highly-trained gynecological surgeons," comments Obermair.

Survival results from these two trials have not yet been published. These data, when available, together with the results of the present studies, will hopefully assist decision making about the introduction of a new surgical technique for the treatment of endometrial cancer.

Lisa Richards

\footnotetext{
Original articles Mourits, M. J. et al. Safety of laparoscopy versus laparotomy in early-stage endometrial cancer: a randomised trial. Lancet Oncol. 11, 763-771 (2010) | Janda, M. et al. Quality of life after total laparoscopic hysterectomy versus total abdominal hysterectomy for stage I endometrial cancer (LACE): a randomised trial. Lancet Oncol. 11, 772-780 (2010)
} 